\title{
Oxytocin enhances the perception of biological motion in humans
}

\author{
SZABOLCS KéRI \\ Semmelweis University, Budapest, Hungary \\ AND \\ GYÖRGY BENEDEK \\ University of Szeged, Szeged, Hungary
}

\begin{abstract}
Evidence suggests that intranasally administered oxytocin modulates several social cognitive and emotional processes in humans. In this study, we investigated the effect of oxytocin on the perception of biological motion (a walking character) and nonbiological motion (a rotating shape). The participants were 20 healthy volunteers who observed moving dots embedded among a cloud of noise (mask) dots. Sensitivity ( $d^{\prime}$ ) for motion detection was determined after the administration of oxytocin and placebo. The results showed that oxytocin (relative to placebo) administration increased sensitivity to biological motion but not to nonbiological motion. These results suggest that oxytocin specifically modulates the perception of socially relevant stimuli.
\end{abstract}

Oxytocin is implicated in a wide range of human social cognitive and emotional functions (Lee, Macbeth, Pagani, \& Young, 2009; Meyer-Lindenberg, 2008). This evolutionarily ancient neuropeptide facilitates the recognition of complex mental states and social emotions (Domes, Heinrichs, Michel, Berger, \& Herpertz, 2007), improves memory for faces (Rimmele, Hediger, Heinrichs, \& Klaver, 2009), increases gaze to the eye regions of other people's faces (Guastella, Mitchell, \& Dadds, 2008), enhances the encoding of positive social memories (Guastella, Mitchell, \& Mathews, 2008) and the recognition of positive sex and relationship words (Unkelbach, Guastella, \& Forgas, 2008), and modulates trust, trustworthiness, and generosity during interpersonal transactions (Baumgartner, Heinrichs, Vonlanthen, Fischbacher, \& Fehr, 2008; Kéri, Kiss, \& Kelemen, 2009; Kosfeld, Heinrichs, Zak, Fischbacher, \& Fehr, 2005; Zak, Kurzban, \& Matzner, 2005; Zak, Stanton, \& Ahmadi, 2007). Oxytocin affects the activation of brain areas responsible for emotion regulation and cognitive control, including the amygdala and the prefrontal cortex (Baumgartner et al., 2008; Domes, Heinrichs, Gläscher, et al., 2007; Kirsch et al., 2005). The genetic polymorphism of the oxytocin receptor gene is associated with prosocial fund allocations in the dictator game and in the social value orientations task (Israel et al., 2009).

In the present study, we investigated the effect of externally administered oxytocin on the visual perception of social and nonsocial stimuli. This question is especially important because the literature is not fully consistent. As discussed above, there is evidence that oxytocin influences gaze to relevant social stimulus details (Guastella, Mitch- ell, \& Dadds, 2008), but, in another study, oxytocin did not affect either gaze toward or the early perceptual detection of threatening and/or positive face stimuli (Guastella, Carson, Dadds, Mitchell, \& Cox, 2009). The perception of biological motion is one of the most fundamental aspects of social cognitive processes and may serve as a primitive "life detector" (Troje \& Westhoff, 2006). The recognition of movements and actions of other people is necessary for communicating, decoding "body language," and learning by imitation (Giese \& Poggio, 2003; Peuskens, Vanrie, Verfaillie, \& Orban, 2005; Puce \& Perrett, 2003). Biological and nonbiological motion stimuli are differentially processed at the semantic level (Goldberg, Perfetti, \& Schneider, 2006). However, the assessment of biological and nonbiological (mechanic) motion is methodologically challenging and requires the special consideration of task difficulty, visual forms defined by motion, and the level of noise that masks the motion. Hiris (2007) demonstrated that biological motion is easier to detect than unstructured nonbiological motion and that the advantage of biological motion disappears when it is compared with structured nonbiological motion that contains a form. The controlled assessment of the perception of biological and nonbiological stimuli is indispensable for obtaining reliable data. Regarding the effect of oxytocin, the crucial issue is the stage and type of information processing. Does oxytocin influence early stages of perception, or is it important only in higher level social cognitive processes? Can oxytocin modulate general perceptual processing-for example, by improving attention and signal-to-noise ratio-or is it specific to biological stimuli? In this study, we investi- 
gated the hypothesis that oxytocin improves the perception of biological motion but has no effect on the detection of structured nonbiological motion.

\section{METHOD}

\section{Participants}

Twenty healthy volunteers ( 10 female; mean age $=28.3$ years, $S D=7.2$; mean education $=14.5$ years, $S D=3.6$; mean $\mathrm{IQ}=109.6$, $S D=10.4$ ) having no history of neurological, psychiatric, or ophthalmological illnesses participated in the study. Visual acuity was normal or corrected to normal. Each participant gave written informed consent, and the study was approved by the local ethics board.

\section{Procedure}

Psychophysical assessment of motion perception. We selected conditions with equal sensitivity and variance for biological and nonbiological motion from the paradigm of Hiris (2007). Three parameters were taken into consideration: stimulus size, number of mask dots, and type of motion. Stimuli were presented on a ViewSonic monitor controlled by a personal computer. The stimuli consisted of 11 white dots presented against a black background from a viewing distance of $60 \mathrm{~cm}$. The whole presentation area subtended a visual angle of $27^{\circ} \times 34^{\circ}$, whereas the stimulus area subtended $6^{\circ} \times 12^{\circ}$ in the horizontal and vertical direction, respectively. The 11 dots comprising the target stimulus were embedded among clouds of either 176 or 352 dynamic mask dots randomly placed on the stimulus area. Scrambled motion masks were used in both biological and non- biological conditions. By the variation of the number of mask dots, we were able to manipulate the difficulty of the task; that is, fewer mask dots allowed easier detection of the target.

There were two types of stimuli. For biological motion, a treadmill walking pattern was used, whereas for nonbiological motion, a structured rotation target appeared on the screen (Figure 1). These stimuli are illustrated by online movies available at http://journalofvision.org/7/12/4/images/Movie1.mov and http:// journalofvision.org/7/12/4/images/Movie3.mov (Hiris, 2007). We replicated and used these stimuli. Each display consisted of 60 frames of motion (rate $=42.5$ frames per sec), which represented one step-cycle of walking for biological motion, and a rotation of $90^{\circ}$ for nonbiological motion.

The task was to judge whether the target stimulus was present within the mask dots or not by pressing one of two different keys on the computer keyboard $(0=n o, 1=y e s)$. The target was present on half of the trials. Each trial was preceded by the presentation of a fixation point for $500 \mathrm{msec}$. There were 100 trials for each stimulus type (biological vs. nonbiological motion) and level of task difficulty (176 vs. 352 mask dots), resulting in a total of 400 trials. Before the experiment, a practice run was provided for each participant, during which the target stimuli were presented without mask dots. The dependent measure of task performance was sensitivity $\left(d^{\prime}\right)$, which was calculated from hit rates and false alarm rates: $d^{\prime}=$ $z_{\text {hit rate }}-z_{\text {false alarm rate }}$, where $z$ is a score calculated using the standard deviation from the mean.

Oxytocin administration. We used the oxytocin administration protocol of Heinrichs, Baumgartner, Kirschbaum, and Ehlert (2003) and Domes, Heinrichs, Michel, et al. (2007). During the experiment,
Biological motion: A walking character

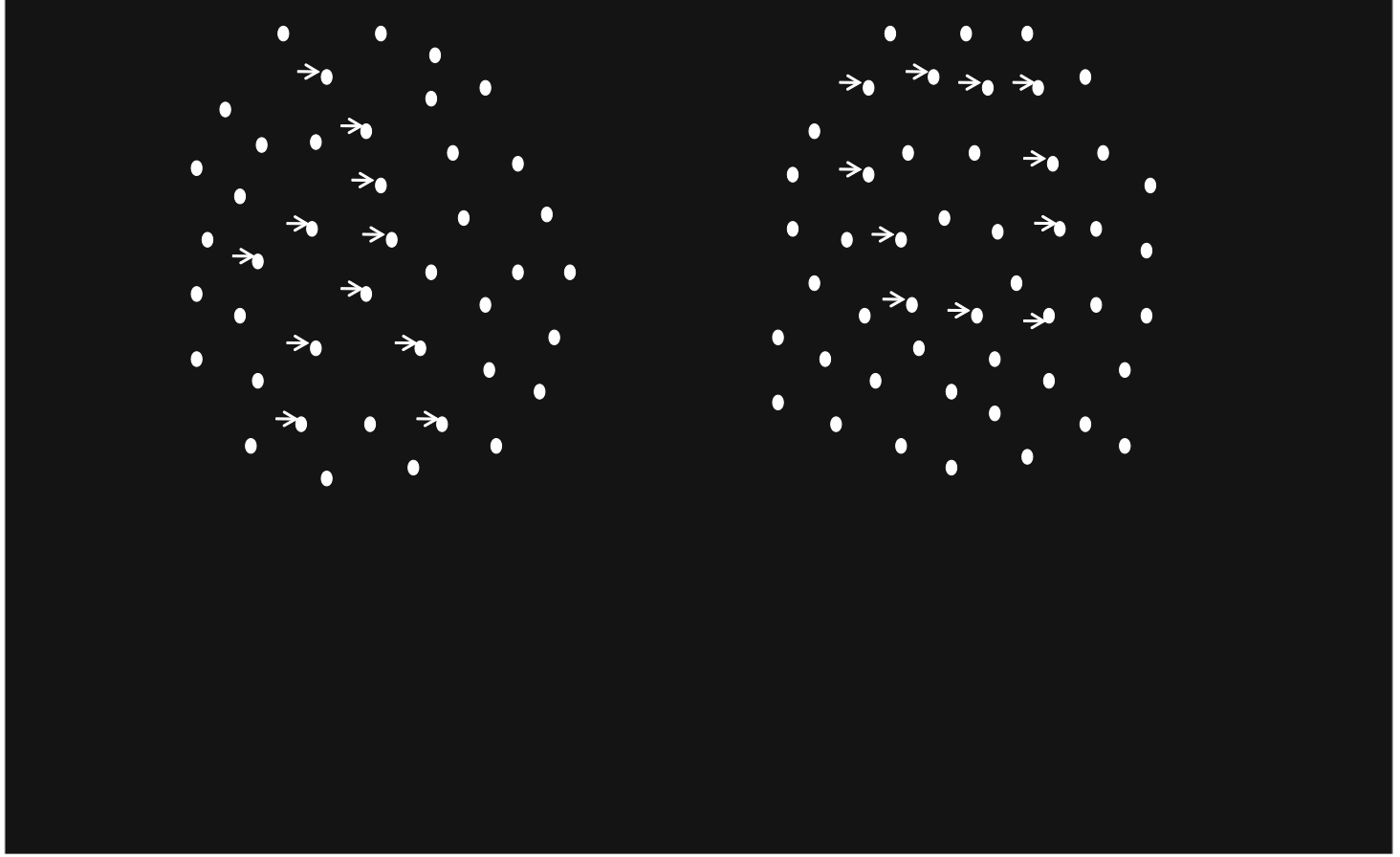

\section{Nonbiological motion: A rotating square}

Figure 1. Illustration of the stimuli used for the assessment of biological and nonbiological motion perception. The walking character performed a treadmill-like motion, whereas the square was rotating (see also the online movies available at http://journalofvision.org/7/12/4/images/Movie1.mov and http://journalofvision.org/7/12/4/images/Movie3.mov [Hiris, 2007]). The signal dots are marked by arrows. 
a single dose of 24 IU oxytocin (Syntocinon spray, Novartis, Basel) or placebo was administered intranasally $45 \mathrm{~min}$ before the beginning of the experiment. Participants underwent both the oxytocin and the placebo conditions with a 1-week interval in a balanced within-subjects design. Participants did not report complaints after the administration of either the oxytocin or the placebo.

\section{Data Analysis}

STATISTICA 7.0 software was used for data analysis (StatSoft, Inc., Tulsa, OK). Levene's tests were used to investigate the homogeneity of variance, and Kolmogorov-Smirnov tests were used to investigate the normality of data distribution. We conducted a repeated measures ANOVA in which $d^{\prime}$ values were the dependent variable and stimulus type (biological vs. nonbiological motion), task difficulty (176 vs. 352 mask dots), and test condition (oxytocin vs. placebo) were the independent variables. $F$ tests were used for planned comparisons, and Scheffé's tests were used for post hoc analysis. The level of significance was set at alpha $<.05$.

\section{RESULTS}

The $d^{\prime}$ values are shown in Figure 2. Levene's tests indicated that the variances for different stimulus types (biological vs. nonbiological) and difficulty levels were homogeneous $(F<0.3, p>.5)$. The Kolmogorov-Smirnov tests indicated that the data did not deviate from the normal distribution $(d<.2, p>.2)$.

The ANOVA conducted on the $d^{\prime}$ scores indicated significant main effects of stimulus type $[F(1,38)=$ $21.09, p<.001, \eta^{2}=.36$, power $\left.=.99\right]$ and task difficulty $\left[F(1,38)=144.92, p<.001, \eta^{2}=.79\right.$, power $=$ 1.0]. The main effect of test condition did not reach the level of statistical significance $[F(1,38)=2.43, p=.13$, $\eta^{2}=.06$, power $\left.=.33\right]$. However, there was a significant two-way interaction between test condition and stimulus type $\left[F(1,38)=30.53, p<.001, \eta^{2}=.45\right.$, power $\left.=.99\right]$. There were no significant interactions between test condition and task difficulty $\left[F(1,38)=0.01, p=.94, \eta^{2}=\right.$ .00 , power $=.05]$ or stimulus type and task difficulty $\left[F(1,38)=0.39, p=.54, \eta^{2}=.01\right.$, power $\left.=.09\right]$ nor among test condition, stimulus type, and task difficulty $\left[F(1,38)=0.46, p=.50, \eta^{2}=.01\right.$, power $\left.=.1\right]$. Scheffé's test conducted on the interaction between test condition and stimulus type revealed that oxytocin increased sensitivity during the perception of biological motion, as compared with the placebo, at both levels of difficulty $(p<.05)$. However, this effect of oxytocin was clearly absent in the case of nonbiological motion $(p>.5)$ (Figure 2). Planned comparisons with $F$ tests indicated that there was no significant difference between the $d^{\prime}$ values for biological and nonbiological motion in the placebo condition $[F(1,38)=0.44, p=.51]$ (Figure 2). In contrast, in the oxytocin condition, biological motion detection was more effective than nonbiological motion detection $[F(1,38)=51.19, p<.001]$, regardless of the level of difficulty.

We also conducted a secondary analysis on participants who had received the placebo first versus those who had received oxytocin first. This ANOVA revealed no significant main effect of order (placebo or oxytocin first) nor any interaction among order, stimulus type, and task difficulty $(F<1, p>.5)$.

\section{DISCUSSION}

A central theme in the literature of motion perception is whether humans are better at detecting biological motion or nonbiological motion (e.g., Casile \& Giese, 2005; Neri,

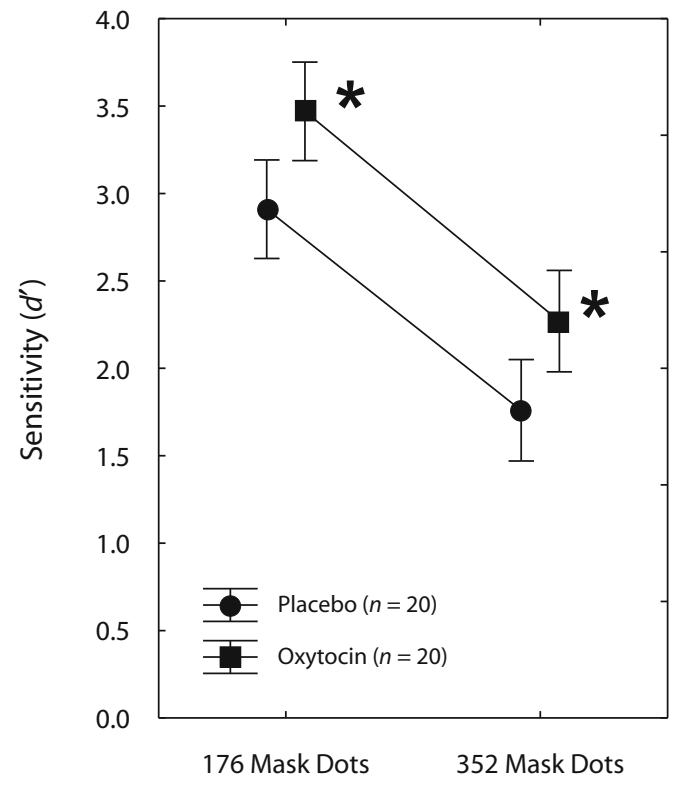

Biological Motion

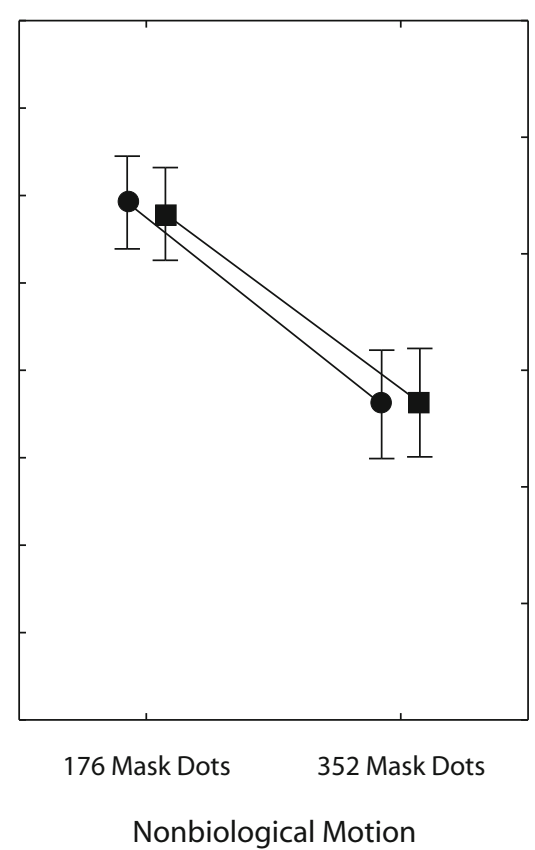

Figure 2. Sensitivity $\left(d^{\prime}\right)$ values for biological and nonbiological motion after intranasal administration of oxytocin and placebo. Data are means. Error bars indicate $95 \%$ confidence intervals. ${ }^{*} p<.05$, Scheffé's tests. 
Morrone, \& Burr, 1998; Pinto \& Shiffrar, 1999; Poom \& Olsson, 2002). In a series of experiments, Hiris (2007) found that any difference of detection efficacy between biological and nonbiological motion could be due to the fact that biological motion always contains an underlying form, whereas nonbiological motion does not necessarily have a form. In the present study, we demonstrated that oxytocin enhances the perception of biological motion by increasing sensitivity for stimuli that represent living objects (a walking character) but does not change the sensitivity for nonbiological stimuli (a rotating square). It is important that we used a psychophysically controlled paradigm (Hiris, 2007) in order to exclude the possibility that the effect of oxytocin might reflect nonspecific factors, such as improved attention or reduced stress related to the challenging task. The differential effect on the biological motion task argues against this possibility. Beyond the interaction between stimulus type and oxytocin administration, no other effects or interactions - including the three-way interaction-were significant. We did not find evidence that participants who received oxytocin in the first session showed better $d^{\prime}$ at the placebo condition than did those who had received the placebo first. This result does not support learning or transfer effects in the present design.

The most important finding of this study is that oxytocin is not only able to modulate higher level social cognitive functions (e.g., mentalization, trust, and facial processing) and affective processes (Meyer-Lindenberg, 2008), but is also able to enhance basic biological motion perception. Although the task was to simply detect a moving object embedded in noise, and although the participants were not required to make inferences about the intentions, emotions, and other mental states of the character (Dittrich, Troscianko, Lea, \& Morgan, 1996; Runeson \& Frykholm, 1983), it cannot be excluded that participants spontaneously made such inferences. This higher level social information may have an impact on perceptual decision making (Mojzisch \& Krug, 2008). Another possibility is that oxytocin allocates the spotlight of attention to biologically relevant stimulus details. Guastella, Mitchell, and Dadds (2008) found that participants given oxytocin showed an increased number of fixations and total gaze time toward the eye region of other people than did placebo participants. Troje and Westhoff (2006) suggested that the enhanced detection of biological motion could be explained by the weighted processing of local limb movements. They proposed a visual filter that is tuned to the characteristic motion of the limbs of humans or of animals in locomotion. It is tempting to speculate that oxytocin may modulate this visual filter and may serve not only as an "eye-region enhancer" but also as a "moving-limb enhancer." From an evolutionary point of view, it is an extremely important function, enabling us to efficiently detect living and moving organisms in our environment.

There is evidence that biological motion activates specific cortical areas (Giese \& Poggio, 2003; Peuskens et al., 2005; Puce \& Perrett, 2003) that also participate in other functions, including mentalization and the processing of social emotions. Data from animal single-cell stud- ies and human functional neuroimaging indicate that the superior temporal sulcus (STS) shows neural responses to moving natural images of faces and bodies, as well as to motion defined by point lights marking the limb articulations of animate bodies. These data are consistent with results from patients with focal brain damage in this area (Saygin, 2007). In addition, the left posterior STS can differentiate between intended and unintended motion (Morris, Pelphrey, \& McCarthy, 2008). We hypothesize that oxytocin has a specific effect on brain areas that participate in biological motion perception; it has been shown that these areas are implicated in higher level social cognition, emotion regulation, attachment, and reward (Baumgartner et al., 2008; Domes, Heinrichs, Gläscher, et al., 2007; Kirsch et al., 2005; for a review of animal functional imaging studies, see Ferris, 2008). In contrast, brain areas (MT/V5 complex) related to the perception of nonbiological motion may be unresponsive to oxytocin. It is important to emphasize that the STS plays a role in multiple social cognitive functions beyond biological motion detection, including face perception, speech processing, and mentalization. The functional role of the STS depends on the pattern of network coactivation with different regions in the frontal cortex and medial-temporal lobe (Hein \& Knight, 2008). These roles of the STS might be a converging point, because oxytocin modulates facial processing (Rimmele et al., 2009) and mentalization (Domes, Heinrichs, Michel, et al., 2007), in addition to modulating biological motion perception. Carter and Pelphrey (2006) demonstrated that mentalization and the development of biological motion perception are closely related; this is consistent with the data discussed above.

In conclusion, in the present study, we have demonstrated that oxytocin enhances the perception of biological motion without any significant effect on the perception of nonbiological motion. Further studies are warranted for the investigation of how oxytocin modulates the activation of sensory and higher cortical areas that are differentially implicated in the processing of social and nonsocial stimuli.

\section{AUTHOR NOTE}

Correspondence concerning this article should be addressed to S. Kéri, Department of Psychiatry and Psychotherapy, Semmelweis University, Balassa u. 6., Budapest, H1083, Hungary (e-mail: keriszabolcs@psych .sote.hu).

\section{REFERENCES}

Baumgartner, T., Heinrichs, M., Vonlanthen, A., Fischbacher, U., \& FEHR, E. (2008). Oxytocin shapes the neural circuitry of trust and trust adaptation in humans. Neuron, 58, 639-650.

Carter, E. J., \& Pelphrey, K. A. (2006). School-aged children exhibit domain-specific responses to biological motion. Social Neuroscience, 1, 396-411.

Casile, A., \& Giese, M. A. (2005). Critical features for the recognition of biological motion. Journal of Vision, 5, 348-360.

Dittrich, W. H., Troscianko, T., Lea, S. E. G., \& Morgan, D. (1996). Perception of emotion from dynamic point-light displays represented in dance. Perception, 25, 727-738.

Domes, G., Heinrichs, M., Gläscher, J., Büchel, C., Braus, D. F., \& Herpertz, S. C. (2007). Oxytocin attenuates amygdala responses to emotional faces regardless of valence. Biological Psychiatry, 62, 1187-1190.

Domes, G., Heinrichs, M., Michel, A., Berger, C., \& Herpertz, 
S. C. (2007). Oxytocin improves "mind-reading" in humans. Biological Psychiatry, 61, 731-733.

FERrIs, C. F. (2008). Functional magnetic resonance imaging and the neurobiology of vasopressin and oxytocin. Progress in Brain Research, 170, 305-320.

Giese, M. A., \& Poggio, T. (2003). Neural mechanisms for the recognition of biological movements. Nature Reviews Neuroscience, $\mathbf{4}$, 179-192.

Goldberg, R. F., Perfetti, C. A., \& Schneider, W. (2006). Distinct and common cortical activations for multimodal semantic categories. Cognitive, Affective, \& Behavioral Neuroscience, 6, 214-222.

Guastella, A. J., Carson, D. S., Dadds, M. R., Mitchell, P. B., \& Cox, R. E. (2009). Does oxytocin influence the early detection of angry and happy faces? Psychoneuroendocrinology, 34, 220-225.

Guastella, A. J., Mitchell, P. B., \& Dadds, M. R. (2008). Oxytocin increases gaze to the eye region of human faces. Biological Psychiatry, 63, 3-5.

Guastella, A. J., Mitchell, P. B., \& Mathews, F. (2008). Oxytocin enhances the encoding of positive social memories in humans. Biological Psychiatry, 64, 256-258.

HeIn, G., \& KNIGHT, R. T. (2008). Superior temporal sulcus-It's my area: Or is it? Journal of Cognitive Neuroscience, 20, 2125-2136.

Heinrichs, M., Baumgartner, T., Kirschbaum, C., \& Ehlert, U. (2003). Social support and oxytocin interact to suppress cortisol and subjective responses to psychosocial stress. Biological Psychiatry, 54, 1389-1398.

HIRIS, E. (2007). Detection of biological and nonbiological motion. Journal of Vision, 7(12, Art. 4), 1-16.

Israel, S., Lerer, E., Shalev, I., Uzefovsky, F., Riebold, M., LAIBA, E., ET AL. (2009). The oxytocin receptor (OXTR) contributes to prosocial fund allocations in the dictator game and the social value orientations task. PLoS ONE, 4, e5535.

KÉRI, S., KISS, I., \& KelEmen, O. (2009). Sharing secrets: Oxytocin and trust in schizophrenia. Social Neuroscience, 4, 287-293.

Kirsch, P., Esslinger, C., Chen, Q., Mier, D., Lis, S., Siddhanti, S., ET AL. (2005). Oxytocin modulates neural circuitry for social cognition and fear in humans. Journal of Neuroscience, 25, 11489-11493.

Kosfeld, M., Heinrichs, M., ZaK, P. J., Fischbacher, U., \& Fehr, E. (2005). Oxytocin increases trust in humans. Nature, 435, 673-676.

Lee, H.-J., Macbeth, A. H., Pagani, J. H., \& Young, W. S. (2009). Oxytocin: The great facilitator of life. Progress in Neurobiology, 88, 127-151.

Meyer-Lindenberg, A. (2008). Impact of prosocial neuropeptides on human brain function. Progress in Brain Research, 170, 463-470.
Mojzisch, A., \& Krug, K. (2008). Cells, circuits, and choices: Social influences on perceptual decision making. Cognitive, Affective, \& Behavioral Neuroscience, 8, 498-508.

Morris, J. P., Pelphrey, K. A., \& McCarthy, G. (2008). Perceived causality influences brain activity evoked by biological motion. Social Neuroscience, 3, 16-25.

Neri, P., Morrone, M. C., \& Burr, D. C. (1998). Seeing biological motion. Nature, 395, 894-896.

Peuskens, H., Vanrie, J., Verfaillie, K., \& Orban, G. A. (2005). Specificity of regions processing biological motion. European Journal of Neuroscience, 21, 2864-2875.

Pinto, J., \& Shiffrar, M. (1999). Subconfigurations of the human form in the perception of biological motion displays. Acta Psychologica, 102, 293-318.

Poom, L., \& Olsson, H. (2002). Are mechanisms for perception of biological motion different from mechanisms for perception of nonbiological motion? Perceptual \& Motor Skills, 95, 1301-1310.

Puce, A., \& Perrett, D. (2003). Electrophysiology and brain imaging of biological motion. Philosophical Transactions of the Royal Society $B, \mathbf{3 5 8}, 435-445$.

Rimmele, U., Hediger, K., Heinrichs, M., \& Klaver, P. (2009). Oxytocin makes a face in memory familiar. Journal of Neuroscience, 29, $38-42$.

Runeson, S., \& Frykholm, G. (1983). Kinematic specifications of dynamics as an informational basis for person-and-action perception: Expectation, gender recognition, and deceptive intention. Journal of Experimental Psychology: General, 112, 585-615.

SAYGIN, A. P. (2007). Superior temporal and premotor brain areas necessary for biological motion perception. Brain, 130, 2452-2461.

Troje, N. F., \& Westhoff, C. (2006). The inversion effect in biological motion perception: Evidence for a "life detector"? Current Biology, 16, 821-824.

Unkelbach, C., Guastella, A. J., \& Forgas, J. P. (2008). Oxytocin selectively facilitates recognition of positive sex and relationship words. Psychological Science, 19, 1092-1094.

ZaK, P. J., Kurzban, R., \& Matzner, W. T. (2005). Oxytocin is associated with human trustworthiness. Hormones \& Behavior, 48, 522-527.

Zak, P. J., Stanton, A. A., \& Ahmadi, S. (2007). Oxytocin increases generosity in humans. PLoS ONE, 2, e1128.

(Manuscript received March 5, 2009; revision accepted for publication June 11, 2009.) 